\title{
Identification of NR and EPDM Samples by Means of Thermogravimetric Analysis and Multivariate Methods
}

\author{
J.-R. Riba, Member, IEEE, T. Canals, and R. Cantero
}

\begin{abstract}
Products based on ethylene-propylene-diene m-class (EPDM) and natural rubber (NR) are widely used in a different applications including the automotive industry, heating, ventilation, and air conditioning applications, roofing systems or the construction sector among others. The growing demand of these types of polymeric products has forced rubber industry to implement strict control schemes to ensure the specifications of the final products. The focus of this paper is the identification of different treatment types of NR and EPDM samples without any preceding analytical treatment to carry out a fast and reliable supervision of the vulcanization processes to improve the quality of the final rubber products. To this end the thermogravimetric analysis (TGA) technique is applied in combination with the principal component analysis (PCA) and canonical variate analysis (CVA) multivariate feature extraction methods and the $k$-nearest neighbor $(k-\mathrm{NN})$ classifier. Experimental results prove the suitability of the proposed approach and the potential of the TGA method for a fast supervision of the vulcanization processes. Using the information provided by the TGA technique in association with the PCA + CVA + $k$-NN approach, the system achieved $100 \%$ identification accuracy.
\end{abstract}

Index Terms-Thermogravimetric analysis, multivariate methods, vulcanization, classification, identification.

\section{INTRODUCTION}

$\mathrm{T}$ HERMAL analysis (TA) includes a set of instrumental techniques applied to measure physical or chemical properties of a substance as function of time or temperature. Although different TA techniques exist, the thermogravimetric analysis (TGA) is applied in this paper since it is a simple, fast and low cost instrumental technique [1]. TGA measures the percentage of weight change of the analyzed specimen as a function of temperature (by applying a constant heating rate) or as a function of time (at constant temperature) in a helium, nitrogen or air atmosphere or under vacuum conditions. By this way useful information is

Manuscript received April 27, 2016;

The authors are grateful to the firm SPC-JEVSA SLU (Castellbisbal, Spain) for supplying the NR and EPDM thermogravimetric data used in this work.

J.-R. Riba is with the Electrical Engineering Department, Escola d'Enginyeria d'Igualada, Universitat Politècnica de Catalunya, Igualada, 08700 Spain (e-mail: jordi.riba@eei.upc.edu).

T. Canals and R. Cantero are with the Escola d'Enginyeria d'Igualada, Universitat Politècnica de Catalunya, Igualada, 08700 Spain (e-mail: trinitat.canals@eei.upc.edu; rosa.cantero@eei.upc.edu). obtained, especially at the temperature points at which sharp weight changes are measured. TGA is used in a broad range of applications including analysis of mixtures since their components have different temperature behavior, chemical identification by analyzing specific decomposition patterns, determination of organic/inorganic content in a sample, or oxidation and reduction analyses among others. TGA is commonly applied to analyze specific characteristics of materials such as elastomers and polymers that experiment changes due to loss of volatiles, phase transitions, decomposition or oxidation. TGA is specially indicated to study polymeric materials and offers several advantages as simplicity, accuracy, reliability, low detection limit, low-cost or minimal sample preparation. This technique is very applied in quality control for both qualitative and quantitative analysis. TGA is considered as an environmentally-friendly analytical method, since it only needs some milligrams of sample while avoiding the use of organic solvents [2]. Fig. 1 schematizes the components involved in a thermogravimetric analyzer.

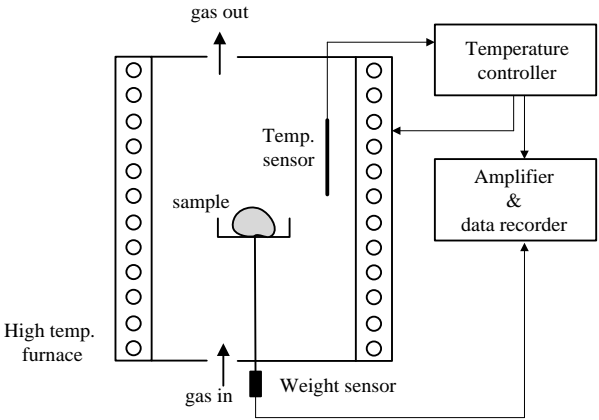

Fig. 1. Diagram of the TGA instrumentation system.

This paper is aimed to improve the supervision and control of rubber processes by identifying differently processed NR and EPDM rubber samples by means of the TGA instrumental technique and the subsequent application of multivariate statistical methods, which include PCA (principal components analysis), CVA (canonical variate analysis) and the $k$-NN ( $k$ nearest neighbor) classifier.

Natural rubber (NR) is a polymeric product derived from latex obtained from the sap of rubber trees although it can be produced synthetically. Uncured NR has few uses, but vulcanized rubber has many applications due to its appealing properties such as elasticity, flexibility, impermeability to gases, resistance to water or high electrical resistivity. 
Vulcanized NR applications include conveyor belts, vehicle tires, pump housings, hoses, balloons, shock absorbers, tubing, electrical insulating gloves, protective shoes, rainwear or diving gear among others.

EPDM rubber is a synthetic elastomeric thermosetting material which offers excellent resistance to water, ozone and heat. EPDM rubber is widely applied in the automotive industry (windows and doors seals, wire harnesses, radiator hoses, etc.), HVAC (heat, ventilation and air conditioning) applications including seals grommets, gaskets and products for pressure tubing, or several industry applications such as belts, electrical insulation, tubbing and water hoses or in the construction sector in cable insulation and roofing systems or profiles among others [3].

It is well-known that uncured NR and EPDM rubber materials present poor mechanical properties, so a vulcanization treatment process is often applied to improve their properties [4]. This process modifies the inner structure by developing crosslinks amid individual chains of polymer, tying the polymer molecules together [5]. As a consequence, the mechanical properties are greatly improved since elasticity is increased and the inherent plasticity of the uncured raw material is significantly reduced [6].

During the vulcanization process reagents and inert products are added to the elastomer. Curatives, a special type of reagents that are added during the compounding process, tend to form bridges that tie the polymer chains in the rubber all together, thus accelerating the crosslinking of the polymeric chains. The most commonly used vulcanizing agent is sulfur jointly with $\mathrm{ZnO}$ and accelerators although a less applied vulcanizing process uses peroxide crosslinking agents combined with co-agents, which allow increasing the yield of crosslinks [4], [7], [8].

Because of the diversity and increasing applications and demand of elastomers, it is necessary to develop accurate and economical control methods intended for the production processes. At present, diverse analytical methods are being applied for controlling the vulcanization processes although such analytical methods are usually time-demanding, involve an individualized interpretation of the results, consume chemicals and reagents and are expensive since they require specialized technicians and laboratory-grade facilities [9][13].

Diverse applications of thermogravimetric techniques for the characterization of rubbers are found in the technical literature. Lah et al. [14] developed a kinetic model based on the application of TGA and DSC (differential scanning calorimetry) to characterize tyre rubber scraps after being subjected to a pyrolytic process. Lopez et al. [15] studied tyre rubber scraps which were subjected to pyrolysis under vacuum conditions by applying both thermogravimetric (TG) and differential thermogravimetric (DTG) techniques. Thermogravimetric analysis was also applied in [16] to determine the composition of waste tyres by developing a kinetic model based on the interactions among the devolatilisation of the distinct rubbers already present in the composition. Yang et al. [17] applied DTG-based methods to qualitatively identify different elastomeric samples.

TGA combined with multivariate techniques has been seldom studied. In [18] different instrumental methods including TGA and different spectroscopic techniques were combined in a data fusion approach to identify beer varieties. A similar approach was applied in [19] to discriminate three almond cultivars based on data provided by TGA, infrared spectroscopy and differential scanning calorimetry.

This work is aimed to develop a direct, fast and accurate method for identifying and classifying differently processed NR and EPDM samples from the thermogravimetric (TG) and differential thermogravimetric (DTG) data without any preceding analytical treatment and without the need to couple other analytical techniques to extract the desired information from the analyzed samples [20], [21]. After applying different phases of the production process to NR and EPDM samples, that is, without and with the application of vulcanizing agents, NR and EPDM specimens were analyzed by means of the TGA instrumental method whose application is simple since it does not require any sample pretreatment or addition of chemicals and reagents. Since both TG and DTG curves contain thousands of data points, it is necessary to analyze this large data set by applying suitable multivariate statistical methods. They concentrate the relevant analytically information in a small set of latent variables, which also allows minimizing the noise and perturbations present in the raw TG and DTG signals [22]-[26].

Multivariate statistical methods have been successfully applied to post-process spectral acquisitions in many process control applications [23] and to determine the composition of blends of polymers [27]-[31] since they allow an exhaustive mathematical interpretation of the spectral information. In this paper this approach is extended to interpret the TG and DTG data.

The CVA (canonical variate analysis) combined with the PCA (principal component analysis) feature extraction methods were applied to calculate the small set of latent variables to be used to identify and classify unknown NR and EPDM samples. Lastly, the $k-\mathrm{NN}$ ( $k$ nearest neighbor) algorithm was applied to classify the incoming and unknown NR and EPDM specimens into their class or treatment type.

The main contribution of this paper is found in the supervision and control of the production processes of NR and EPDM compounds from the chemometric analysis of the TG and DTG data. Due to the complex composition of the analyzed samples, the identification of such samples results in a challenging problem.

\section{THE ANALYZED SAMPLES}

In this paper a total amount of 60 samples are studied, that is $30 \mathrm{NR}$ and $30 \mathrm{EPDM}$ samples from SPC-JEVSA SLU (Castellbisbal, Spain) specialized in manufacturing custom rubber products. Both the NR and EPDM samples were split into three groups according to the treatment type, namely RS (raw samples), VAS (samples with vulcanizing agents) and 
VS (vulcanized samples) as shown in Table I. Note that NR/EPDM polymers are the main component in specimens conforming class RS although they may also contain carbon black acting as a reinforcing element to infer the final vulcanizing abrasion resistance, resilience and high strength as well as plasticizer additives. EPDM samples in class VAS also incorporate peroxide whereas NR samples in class VAS include sulfur as vulcanizing agent to increase the yield of the crosslinks [4], [7], [8]. Vulcanized samples in class VS were vulcanized by using a press with a $2 \mathrm{~mm}$ thick mold for plates and by heating appropriately.

The total set of rubber samples was randomly divided into two sets, the calibration and prediction sets. Whereas the calibration set was used to adjust or calibrate the multivariate classification model, the prediction set was used to check the accuracy of the model accuracy when classifying NR and EPDM samples which are not included in the calibration set.

\section{DATA ACQUisition By MEANS Of ThERMogRaVIMETRIC ANALYSIS (TGA)}

The thermogravimetric curves of the $30 \mathrm{NR}$ and 30 EPDM samples were acquired by using a thermogravimetric analyzer (Q500, TA Instruments, New Castle, USA), which allows monitoring the weight loss as a function of temperature and evaluating the thermal decomposition of the analyzed samples over time.

Small rubber samples of 5 to $14 \mathrm{mg}$ were placed on a platinum pan. To measure the oil and polymer weight loss, the TG measurements were run under an inert atmosphere of nitrogen gas that prevented the samples from oxidation, followed by a later phase under an air atmosphere to measure the weight loss due to the oxidation of the black-carbon and inorganic components. Experiments were performed under a heating rate of $20^{\circ} \mathrm{C} / \mathrm{min}$ and a gas flow of $90 \mathrm{~mL} / \mathrm{min}$. By this way both TG (thermogravimetric) and DTG (differential thermogravimetric) curves were obtained.

TG and DTG thermograms of elastomers are directly related to their composition. Until approximately $300^{\circ} \mathrm{C}$, the resulting signal is related to highly volatile matter such as monomer leftovers, extenders, processing oils, curatives, plasticizers, or antioxidants. The $300-750^{\circ} \mathrm{C}$ range usually provides information about medium volatile components such as elastomers, processing oils and aids or curing agents among others. Beyond $750^{\circ} \mathrm{C}$, the information obtained is due to oxidizable non-volatile combustible material such as carbonblack or graphite. Information about the ash left after oxidative decomposition of inorganic components can also be obtained [32]. However, in order to collect the most significant information about the elastomers under study, this work deals within the $30.5-600{ }^{\circ} \mathrm{C}$ temperature range.

All TG and DTG curves are composed of 2273 data points, thus the $30 \mathrm{NR}$ samples provided a data matrix consisting of 30 rows (rubber samples) and 2273 columns (relative weight points), the same being valid for the 30 EPDM samples.

Figs. $2 \mathrm{a}$ and $2 \mathrm{~b}$ show, respectively, the TG and DTG curves of the 15 NR samples included in the calibration set. Despite their similitude, some weight loss differences can be appreciated in both types of curves among RS, VAS and VS samples. Most representative temperatures are found around $170^{\circ} \mathrm{C}$, because of the weight loss due to the volatile products and water, around $250^{\circ} \mathrm{C}$ due to the oil and at $388^{\circ} \mathrm{C}$ because of the pyrolysis of the NR polymer.

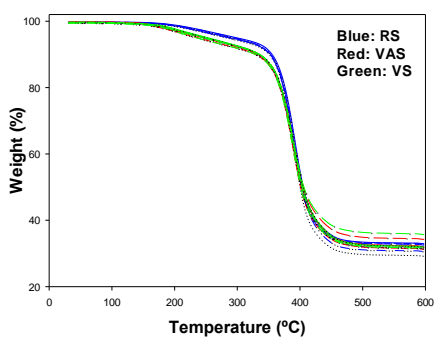

a)

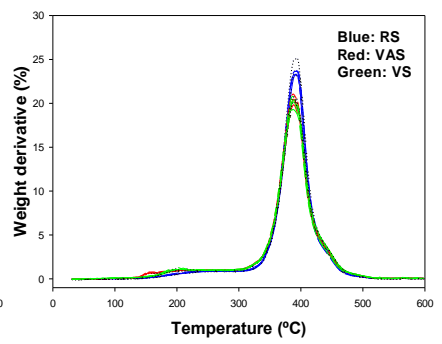

b)
Fig. 2. a) TG curve expressing the weight (\%) against temperature $\left({ }^{\circ} \mathrm{C}\right)$ of the 15 calibration NR samples. b) Differential TG (DTG) curve of the same NR samples.

Figs. 3a and $3 b$ show, respectively, the TG and DTG curves of the 15 EPDM samples included in the calibration set within the temperature range $30.5^{\circ} \mathrm{C}-600^{\circ} \mathrm{C}$. Both type of curves show similar behavior although a minor weight loss difference is observed among RS, VAS and VS samples. Temperature values with more pronounced weight loss are $293^{\circ} \mathrm{C}$ due to the volatile products and $478^{\circ} \mathrm{C}$ because of the pyrolysis of the EPDM polymer.

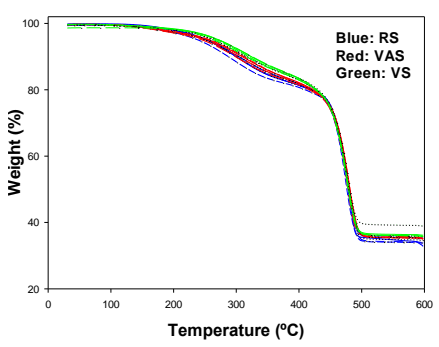

a)

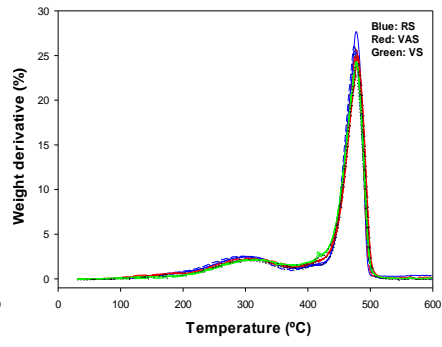

b)
Fig. 3. a) TG curve expressing the weight (\%) against temperature $\left({ }^{\circ} \mathrm{C}\right)$ of the 15 calibration EPDM samples. b) Differential TG (DTG) curve of the same EPDM samples.

By comparing the DTG curves it is clearly appreciated that the main peak is displaced at $378^{\circ} \mathrm{C}$ in the NR-DTG curve and around $478^{\circ} \mathrm{C}$ in the EPDM-DTG curve. The secondary peak exhibits a similar behavior, as it is shifted from around $200{ }^{\circ} \mathrm{C}$ to $300{ }^{\circ} \mathrm{C}$ since EPDM elastomers exhibit more resistance to temperature and outdoor conditions than NR elastomers as shown in the comparative results displayed in Figs. 4.

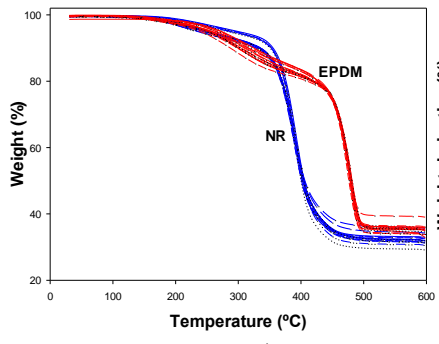

a)

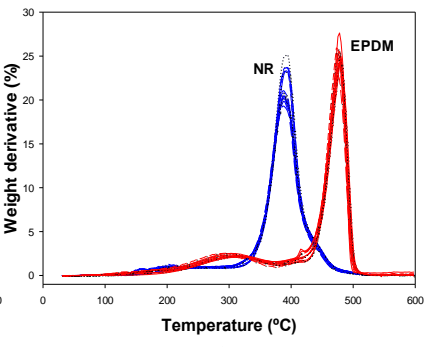

b)
Fig. 4. Comparison of NR and EPDM samples. a) TG curves. b) DTG curves. 


\section{FEATURE EXTRACTION AND ClassificATION METHODS}

The TG and DTG curves of each rubber sample as provided by the TGA instrument consists of 2273 data points (relative weights as a function of temperature for the TG curves or the temperature derivative of the weight for the DTG curves). When dealing with datasets containing a large number of variables, it becomes imperative to deal with fast and efficient multivariate processing methods which allow concentrating the analytically significant information in a reduced set of latent variables [23], [25]. Multivariate feature extraction methods calculate a reduced set of latent variables from a large set of original variables, while eliminating most of the noise often present in the original signals. Supervised feature extraction methods are often preferred over unsupervised methods, since the first ones use class labels to assign the samples to the classes defined in the problem. The class labels of the samples in the calibration set are defined by an expert user, who guides the supervised classification algorithm, which enhances discrimination among classes [25]. Feature extraction algorithms often calculate the latent variables by combining the original variables.

CVA is one of the most powerful supervised multi-class feature extraction methods [25], [33] which was conceived to maximize separation among classes [34]. To accentuate separation among classes, CVA explores the directions in the space defined by the original variables that boost the separation among classes and simultaneously minimize the variability of the samples within classes. Canonical variates or $\mathrm{CVs}$, are the non-orthogonal latent variables obtained by applying the CVA algorithm based on the Fisher's criterion [35]. The number of CVs provided by the CVA algorithm equals the number of classes defined in the problem minus one. However, CVA requires data sets containing more samples than variables, this being an important disadvantage of this multivariate method. This is not the case of the problem under analysis, in which there are 2273 original variables and only 30 samples. To circumvent this drawback, the PCA algorithm is applied to reduce the number of variables prior application of the CVA [22]. PCA is amongst the most widely applied unsupervised feature extraction methods [25], [36], [37]. PCA is aimed to condense the statistically significant information explained by the original variables into a reduced set of orthogonal latent variables called principal components or PCs [35]. The PCA algorithm redistributes the overall variance into the latent variates. The PCs, which denote the perpendicular directions with higher variance, are obtained by applying linear combinations of the original variables. PCA calculates as many PCs as original variables in the problem and ranks the PCs in decreasing variance order, that is, the first $\mathrm{PC}$ is the one explaining the higher variance whereas the last one explains the lowest variance. Finally, only the first PCs explaining a sufficient amount of the overall variance are retained, the remaining ones being disregarded. By carefully retaining an adequate number of PCs and splitting the overall set of samples into two sets, the calibration and the training set, overfitting can be avoided [38].

The classification step is applied after reducing the number of variables of the problem by means of the PCA+CVA dimensionality reduction approach. To this end, the $k$-NN ( $k$ nearest neighbor) classifier is applied since it is one of the simplest and most effective classifiers [26]. This supervised algorithm is based on the majority vote, since it assigns an unknown incoming sample of the prediction set to the most voted class by considering the weighted vote of the $k$ nearest neighbors in the calibration set. The $k$-NN algorithm assigns a score $k$ to the class of the nearest neighbor in the calibration set, a score $k-1$ to the second nearest neighbor's class and so on until assigning a unity score to the $k$-th nearest neighbor. It has to be noted that $k$-NN usually provides the same number of normalized output values within the interval $[0,1]$ as classes defined in the problem. These normalized output values define the membership degree of the unknown input rubber samples to each of the classes.

\section{RESULTS AND DISCUSSION}

The classification of unknown incoming rubber samples by applying the PCA + CVA $+k-N N$ approach shown in Fig. 5 is carried out in this section.

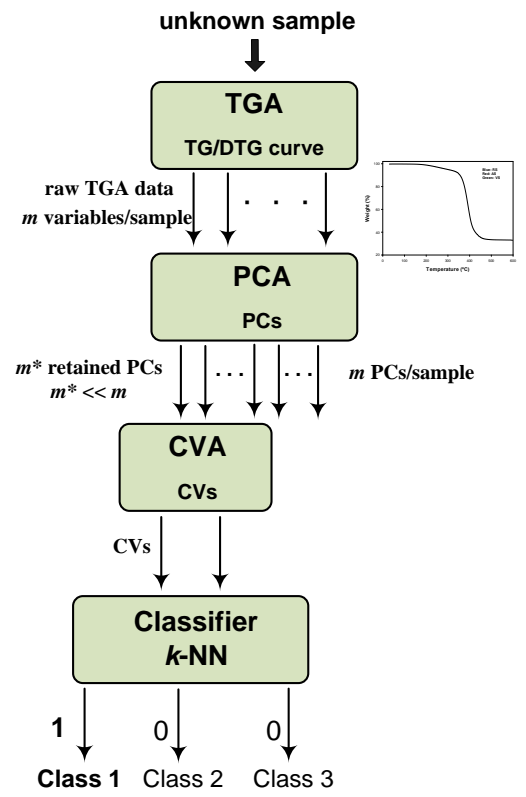

Fig. 5. Flowchart of the PCA + CVA $+k$-NN approach to classify unknown $\mathrm{NR} / \mathrm{EPDM}$ samples according to the type of processing.

It is assumed that the differences among treatment types among samples are reflected in their TG and DTG curves.

In this section the $30 \mathrm{NR}$ and 30 EPDM samples belonging to three classes or treatment types are analyzed and classified by applying the PCA + CVA $+k$-NN approach. The three classes are raw samples (RS), samples with vulcanizing agents (VAS) and vulcanized samples (VS), with 10 samples each. The samples of each treatment type were randomly split into a calibration and a prediction set with the same number of samples each. Table I summarizes the number of samples included in each set.

According to Table I, the data matrixes of the calibration and prediction set samples for both the TG and DTG data consist of $15 \times 2273$ elements. The 2273 columns correspond to the weight-temperature (TG data) or weight-derivative- 
temperature (DTG data), each row corresponding to each sample.

TABLE I

ANALYZED NR AND EPDM RUBBER SAMPLES

\begin{tabular}{ccccc}
\hline \hline Type & Group & $\begin{array}{c}\text { Calibration } \\
\text { set }\end{array}$ & $\begin{array}{c}\text { Prediction } \\
\text { set }\end{array}$ & $\begin{array}{c}\text { Total } \\
\text { samples }\end{array}$ \\
\hline \multirow{4}{*}{ NR } & Raw samples (RS) & 5 & 5 & 10 \\
& Samples with vulcanizing & 5 & 5 & 10 \\
& agents (VAS) & 5 & 5 & 10 \\
& Vulcanized samples (VS) & 5 & $\mathbf{1 5}$ & $\mathbf{3 0}$ \\
\hline \multirow{4}{*}{ EPDM } & Total & $\mathbf{1 5}$ & 5 & 10 \\
& Raw samples (RS) & 5 & 5 & 10 \\
& Samples with vulcanizing & 5 & 5 & 10 \\
& agents (VAS) & 5 & $\mathbf{1 5}$ & $\mathbf{3 0}$ \\
\hline \hline
\end{tabular}

\section{A. NR rubber samples}

Two first studies of the NR samples were carried out using the information from the TG and DTG data. Due to the 2273 data points provided by the TG curves and the reduced number of samples available (15 in both the calibration and prediction sets), it is mandatory to apply the PCA algorithm previous to the application of the CVA feature extraction method. However, this approach requires a selection of a reduced number of PCs arising from the PCA to improve separation and avoid overfitting.

The first study carried on the NR samples was based on the information from the TG curves. The number of PCs explaining more than $99.5 \%$ of the total variance was retained, as shown in Fig. 6, condition that was fulfilled with the first 5 PCs. By this way the problem dimensionality was reduced from 2273 to only 5 PCs thus enabling the posterior application of the CVA algorithm.

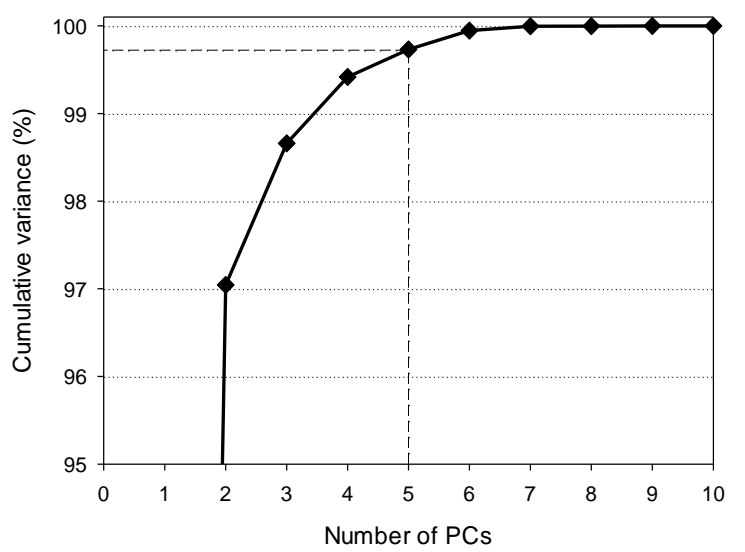

Fig. 6. NR-TG data of the calibration set. Cumulative variance versus the number of PCs retained.

Fig. 7 shows the dispersion plot of both the calibration and prediction sets as obtained from the two CVs arising from the application of the PCA (5 PCs) + CVA approach.

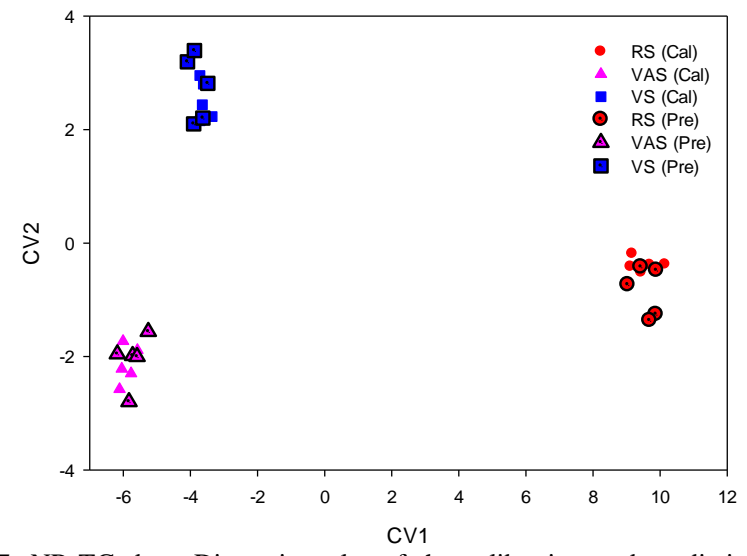

Fig. 7. NR-TG data. Dispersion plot of the calibration and prediction sets considering the three classes (RS, VAS and VS) in the plane of the two CVs arising from the PCA (5 PCs) + CVA.

Next, the $k$-NN algorithm was applied to classify the samples in the prediction set, resulting in 15 correctly classified samples in the prediction set when dealing with $k=$ 3,4 and 5 neighbors, that is, $100 \%$ classification rate in all cases.

A second study of the NR samples was carried out by analyzing the information provided by the DTG curves following the same approach as in the previous study. Fig. 8 shows the cumulative variance curve as a function of the number of PCs retained, which shows that to explain $99.5 \%$ of the total variance, the first 5 PCs are required.

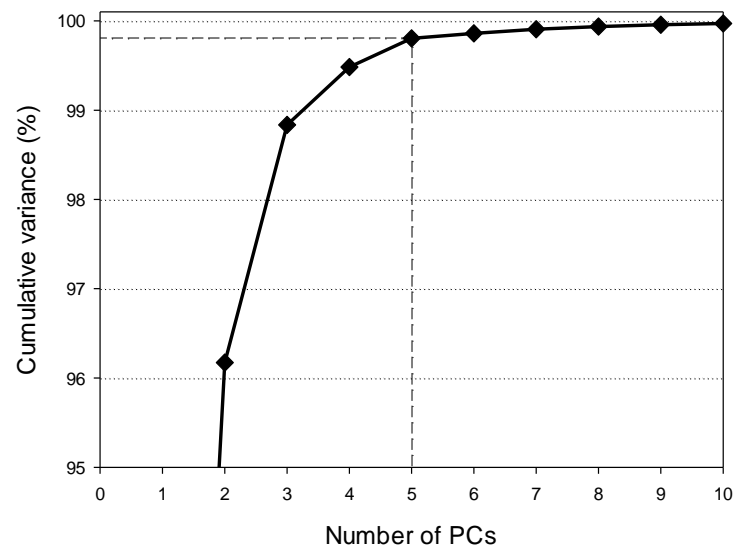

Fig. 8. NR-DTG data of the calibration set. Cumulative variance versus the number of PCs retained.

Fig. 9 shows the dispersion plot of both the calibration and prediction sets as obtained from the two CVs arising from the application of the PCA (5 PCs) + CVA approach. 


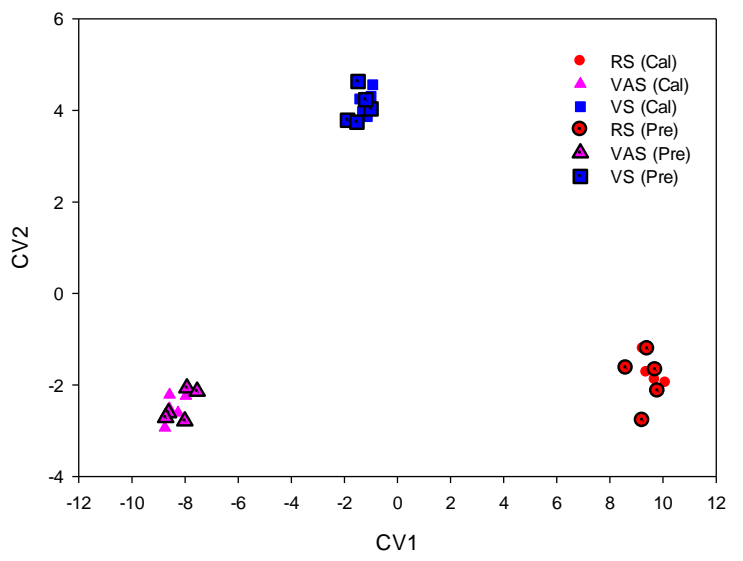

Fig. 9. NR-DTG data. Dispersion plot of the calibration and prediction sets of the three classes (RS, VAS and VS) in the plane of the two CVs arising from the PCA (5 PCs) + CVA.

Next, the $k$-NN algorithm was applied to classify the samples in the prediction set, resulting in 15 correctly classified samples out of the 15 samples in the prediction set when dealing with $k=3,4$, and 5 neighbors, that is $100 \%$ classification rate in all cases.

\section{B. EPDM samples}

Similarly as done with the NR samples, two studies of the EPDM specimens were carried out, the first one by analyzing the data provided by the TG curves, whereas the second study was performed from the data of the DTG curves.

From the first study applied to the EPDM samples, which was based on the information provided by the TG curves, it was found that to explain at least $99.5 \%$ of the total variance, it was necessary to only retain the first 7 PCs. This strategy reduces the dimensionality of the problem from 2273 to only 7 PCs, thus permitting a subsequent application of the CVA feature extraction algorithm.

Fig. 10 shows the dispersion plot of both the calibration and prediction set samples as obtained from the two CVs arising from the application of the PCA (7 PCs) + CVA approach.

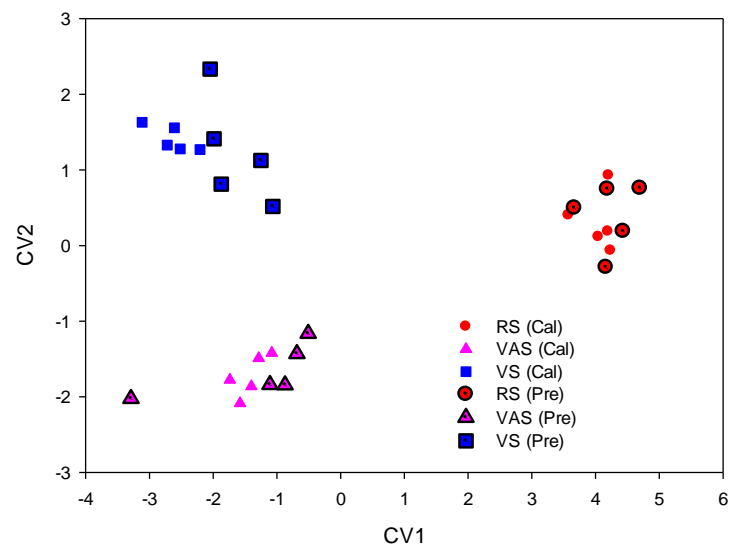

Fig. 10. EPDM-TG data. Dispersion plot of the calibration and prediction sets of the three classes (RS, VAS and VS) in the plane of the two CVs arising from the PCA (7 PCs) + CVA.

Although EPDM samples in Fig. 10 appear more dispersed than NR samples (compare Fig. 10 with Figs. 7 and 9), the samples appear grouped according to their class. The $k$-NN algorithm provides $100 \%$ classification accuracy for all cases $(k=3,4$ and 5$)$.

The second study of the EPDM samples considered the data from the DTG curves by applying the same approach as in the first study. In this case the first 7 PCs allowed explaining more than $99.5 \%$ of the total variance. Fig. 11 displays the dispersion plot of the calibration and prediction sets of EPDM samples in the space of the two CVs obtained from the application of the PCA (7 PCs) + CVA approach.

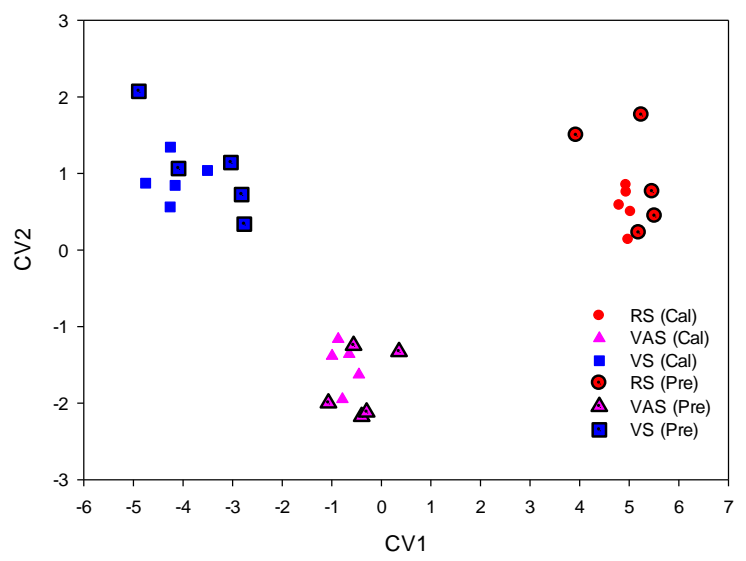

Fig. 11. EPDM-DTG data. Dispersion plot of the calibration and prediction sets of the three classes (RS, VAS and VS) in the plane of the two CVs arising from the PCA (7 PCs) + CVA.

Once again, the $k$-NN algorithm was applied to classify the samples in the prediction set. It resulted in $100 \%$ classification rate accuracy of the samples in the prediction set when dealing with $k=3,4$, and 5 .

\section{Results summary}

Table II summarizes the results attained in this paper for the NR and EPDM samples when dealing with both TG and DTG data.

TABLE II

RESULTS SUMMARY

\begin{tabular}{cccc}
\hline $\begin{array}{c}\text { Feature extraction } \\
\text { methods }\end{array}$ & $\begin{array}{c}\text { Data } \\
\text { curve }\end{array}$ & $\begin{array}{c}\boldsymbol{k} \text {-NN } \\
\text { classifier }\end{array}$ & $\begin{array}{c}\text { Prediction } \\
\text { success rate }\end{array}$ \\
\hline & NR samples & \\
\hline & & $k=3$ & $15 / 15(100 \%)$ \\
PCA (5 PCs) + CVA & TG & $k=4$ & $15 / 15(100 \%)$ \\
& & $k=5$ & $15 / 15(100 \%)$ \\
\hline & & $k=3$ & $15 / 15(100 \%)$ \\
PCA (5 PCs) + CVA & DTG & $k=4$ & $15 / 15(100 \%)$ \\
& & $k=5$ & $15 / 15(100 \%)$ \\
\hline & EPDM samples & \\
\hline & & $k=3$ & $15 / 15(100 \%)$ \\
PCA (7 PCs) + CVA & TG & $k=4$ & $15 / 15(100 \%)$ \\
& & $k=5$ & $15 / 15(100 \%)$ \\
\hline & & $k=3$ & $15 / 15(100 \%)$ \\
PCA (7 PCs) + CVA & DTG & $k=4$ & $15 / 15(100 \%)$ \\
& & $k=5$ & $15 / 15(100 \%)$ \\
\hline \hline
\end{tabular}

It is worth noting that the $k$-NN classifier produces as many output values as classes defined in the problem, whose values are normalized in the $[0,1]$ interval. The $[0,1]$ values describe the membership degree of the considered sample to each class, so an incoming unknown sample is assigned to the class whose output is greater than 0.5. However, in order to avoid uncertainties, values close to 1 are preferred to those close to 
0.5 to be sure that the sample belongs to the considered class. In all cases summarized in Table II, the output values of all samples were either 0 or 1 , thus indicating absolute certainty of the results attained.

\section{CONCLUSION}

In this paper an approach based on the TGA instrumental technique combined with a multivariate statistical analysis has been presented to discriminate differently processed NR and EPDM rubber samples. It is a simple, fast, low cost and environmentally-friendly instrumental technique since the samples do not require any previous analytical treatment, thus avoiding laboratory grade facilities and the need of a skilled technician. To this end, data from TG and DTG curves provided by the TGA instrumental method have been processed by means of the PCA and CVA algorithms in order to extract a reduced set of relevant latent variables which are the input of the $k$-NN classifier. Experimental results reported in this paper verify that for the analyzed rubber samples, which include different treatments, the proposed approach based on the data provided by the TGA instrumental technique and the PCA $+\mathrm{CVA}+k$-NN classification approach allows a fast and very accurate identification of the different treatments. The accurate and reliable results presented in this paper show the potential of the proposed approach in the supervision and control of the vulcanization processes of NR and EPDM compounds from the statistical analysis of the TG and DTG curves, thus it can be very useful for the rubber industry.

\section{REFERENCES}

[1] J. F. Saldarriaga, R. Aguado, A. Pablos, M. Amutio, M. Olazar, and J. Bilbao, "Fast characterization of biomass fuels by thermogravimetric analysis (TGA)," Fuel, vol. 140, pp. 744-751, Jan. 2015.

[2] M. Khanmohammadi, M. Soleimani, F. Morovvat, A. B. Garmarudi, M. Khalafbeigi, and K. Ghasemi, "Simultaneous determination of paracetamol and codeine phosphate in tablets by TGA and chemometrics," Thermochim. Acta, vol. 530, pp. 128-132, Feb. 2012.

[3] K. A. J. Dijkhuis, "Recycling of vulcanized EPDM rubber," University of Twente, 2008.

[4] M. A. Mansilla, "Influence of the microstructure on the mechanical an thermal properties of Natural Rubber and Styrene Butadiene Rubber," Facultad de Ciencias Exactas y Naturales. Universidad de Buenos Aires, 2012

[5] J. E. Mark and B. Erman, Science and Technology of Rubber. 1994.

[6] S. Flanderijn, "Mechanochemical devulcanization of butadiene rubber with DPDS as a devulcanization aid." 27-Jul-2012.

[7] J. E. Kogel, Industrial Minerals \& Rocks: Commodities, Markets, and Uses. SME, 2006.

[8] J. P. J. Zhao, G. Ghebremeskel, "Properties of EPDM/SBR Blends Cured with Peroxide and Sulfur Coagent," Elastomers Plast., vol. 5, pp. 223-228, 2001.

[9] D. Hirayama and C. Saron, "Chemical Modifications in StyreneButadiene Rubber after Microwave Devulcanization," Ind. Eng. Chem. Res., vol. 51, no. 10, pp. 3975-3980, Mar. 2012.

[10] C. Tzoganakis and Q. Zhang, "Devulcanization of Recycled Tire Rubber Using Supercritical Carbon Dioxide | Society of Plastics Engineers," in Global Plastics Environmental Conference, 2004, pp. 111.

[11] N. V. Baranova, L. A. Pashina, G. G. Suchkova, and A. V. Kostochko, "Chemical composition of the surface of modifying polymers in filled cellulose nitrate composites: An IR study," Russ. J. Appl. Chem., vol. 85, no. 10, pp. 1600-1609, Nov. 2012.

[12] B. Maridass and B. R. Gupta, "Recycling of Waste Tire Rubber
Powder. Devulcanization in a Counter Rotating Twin Screw Extruder," KGK Kautschuk Gummi Kunststoffe, vol. 56, no. 5, pp. 232-236, 2003.

[13] S. Saiwari, "Post-consumer tires back into new tires: de-vulcanization and re-utilization of passenger car tires," University of Twente, Enschede, the Netherlands, 2013.

[14] B. Lah, D. Klinar, and B. Likozar, "Pyrolysis of natural, butadiene, styrene-butadiene rubber and tyre components: Modelling kinetics and transport phenomena at different heating rates and formulations," Chem. Eng. Sci., vol. 87, pp. 1-13, 2013.

[15] G. Lopez, R. Aguado, M. Olazar, M. Arabiourrutia, and J. Bilbao, "Kinetics of scrap tyre pyrolysis under vacuum conditions," Waste Manag., vol. 29, no. 10, pp. 2649-2655, 2009.

[16] B. Danon and J. Görgens, "Determining rubber composition of waste tyres using devolatilisation kinetics," Thermochim. Acta, vol. 621, pp. $56-60,2015$

[17] J. Yang, S. Kaliaguine, and C. Roy, "Improved Quantitative Determination of Elastomers in Tire Rubber by Kinetic Simulation of DTG Curves," http://dx.doi.org/10.5254/1.3538307, 2012.

[18] A. Biancolillo, R. Bucci, A. L. Magrì, A. D. Magrì, and F. Marini, "Data-fusion for multiplatform characterization of an Italian craft beer aimed at its authentication," Anal. Chim. Acta, vol. 820, pp. 23-31, Apr. 2014

[19] A. V. García, A. Beltrán Sanahuja, and M. del C. Garrigós Selva, "Characterization and classification of almond cultivars by using spectroscopic and thermal techniques.," J. Food Sci., vol. 78, no. 2, pp. C138-44, Feb. 2013

[20] S. Chen, H. Yu, W. Ren, and Y. Zhang, "Thermal degradation behavior of hydrogenated nitrile-butadiene rubber (HNBR)/clay nanocomposite and HNBR/clay/carbon nanotubes nanocomposites," Thermochim. Acta, vol. 491, no. 1-2, pp. 103-108, Jul. 2009.

[21] N. S. Tomer, F. Delor-Jestin, R. P. Singh, and J. Lacoste, "Crosslinking assessment after accelerated ageing of ethylene propylene diene monomer rubber," Polym. Degrad. Stab., vol. 92, no. 3, pp. 457-463, Mar. 2007.

[22] T. Canals, J. Riba, R. Cantero, J. Cansino, D. Domingo, and H. Iturriaga, "Characterization of paper finishes by use of infrared spectroscopy in combination with canonical variate analysis," Talanta, vol. 77, no. 2, pp. 751-757, Dec. 2008.

[23] J.-R. Riba, T. Canals, R. Cantero, and H. Iturriaga, "Potential of infrared spectroscopy in combination with extended canonical variate analysis for identifying different paper types," Meas. Sci. Technol., vol. 22, no. 2, p. 025601, Feb. 2011.

[24] J.-R. Riba Ruiz, T. Canals, and R. Cantero Gomez, "Comparative Study of Multivariate Methods to Identify Paper Finishes Using Infrared Spectroscopy," IEEE Trans. Instrum. Meas., vol. 61, no. 4, pp. 1029-1036, Apr. 2012.

[25] J.-R. Riba, T. Canals, and R. Cantero, "Recovered Paperboard Samples Identification by Means of Mid-Infrared Sensors," IEEE Sens. J., vol. 13, no. 7, pp. 2763-2770, Jul. 2013.

[26] J.-R. Riba, T. Canals, and R. Cantero, "Determination of the recoveredfiber content in paperboard samples by applying mid-infrared spectroscopy.," Appl. Spectrosc., vol. 69, no. 4, pp. 442-50, Apr. 2015.

[27] D. M. Stelescu, A. Airinei, M. Homocianu, N. Fifere, D. Timpu, and M. Aflori, "Structural characteristics of some high density polyethylene/EPDM blends," Polym. Test., vol. 32, no. 2, pp. 187-196, Apr. 2013.

[28] C. E. Miller, B. E. Eichinger, T. W. Gurley, and J. G. Hermiller, "Determination of microstructure and composition in butadiene and styrene-butadiene polymers by near-infrared spectroscopy," Anal. Chem., vol. 62, no. 17, pp. 1778-1785, Sep. 1990.

[29] A. J. Durbetaki and C. M. Miles, "Near Infrared and Nuclear Magnetic Resonance Spectrometry in Analysis of Butadiene Polymers.," Anal. Chem., vol. 37, no. 10, pp. 1231-1235, Sep. 1965.

[30] F. Vilmin, C. Dussap, and N. Coste, "Fast and robust method for the determination of microstructure and composition in butadiene, styrenebutadiene, and isoprene rubber by near-infrared spectroscopy.," Appl. Spectrosc., vol. 60, no. 6, pp. 619-30, Jun. 2006.

[31] Y. Sulub and J. DeRudder, "Determination of polymer blends composed of polycarbonate and rubber entities using near-infrared (NIR) spectroscopy and multivariate calibration," Polym. Test., vol. 32, no. 4, pp. 802-809, Jun. 2013.

[32] J. C. J. Bart, Plastics additives: advanced industrial analysis. Amsterdam: IOS Press, 2006.

[33] P.-E. P. Odiowei, "Nonlinear Dynamic Process Monitoring Using Canonical Variate Analysis and Kernel Density Estimations," IEEE 
Trans. Ind. Informatics, vol. 6, no. 1, pp. 36-45, Feb. 2010.

[34] L. Nørgaard, R. Bro, F. Westad, and S. B. Engelsen, "A modification of canonical variates analysis to handle highly collinear multivariate data," J. Chemom., vol. 20, no. 8-10, pp. 425-435, Aug. 2006.

[35] R. A. Johnson and D. W. Wichern, Applied Multivariate Statistical Analysis, 6th editio. Englewood Cliffs, NJ (USA): Prentice-Hall, 2007.

[36] R. Faleh, M. Othman, S. Gomri, K. Aguir, and A. Kachouri, "A transient signal extraction method of WO3 gas sensor array to identify polluant gases," IEEE Sens. J., vol. PP, no. 99, pp. 1-1, 2016.

[37] S. Shokralla, J. Morelli, and T. Krause, "Principal Components Analysis of Multi-frequency Eddy Current Data Used to Measure Pressure Tube to Calandria Tube Gap," IEEE Sens. J., vol. PP, no. 99, pp. 1-1, 2016.

[38] N. Bhattacharyya, R. Bandyopadhyay, M. Bhuyan, B. Tudu, D. Ghosh, and A. Jana, "Electronic Nose for Black Tea Classification and Correlation of Measurements With 'Tea Taster' Marks," IEEE Trans. Instrum. Meas., vol. 57, no. 7, pp. 1313-1321, Jul. 2008.

Jordi-Roger Riba Ruiz (M'09) received the M.S. degree in physics and the Ph.D. degree from Universitat de Barcelona, Barcelona, Spain, in 1990 and 2000, respectively. In 1992, he joined Escola d'Enginyeria d'Igualada, Universitat Politècnica de Catalunya (Barcelona, Spain), as a full-time Lecturer, and he joined the Department of Electric Engineering in 2001. His research interests include signal processing methods, application of chemometrics for process control in different industrial sectors, electromagnetic devices modeling, electric machines, variable-speed drive systems, fault diagnosis in electric machines, and fault detection algorithms.

Trini Canals Parelló obtained the degree in Chemistry at the University of Barcelona (Spain) in 1980 and the Ph.D. degree in Chemistry at the University Autònoma of Barcelona in 1997. She received a Master's in Tanning Technical Management from the Universitat Politècnica de Catalunya (Barcelona, Spain) in 1999. Since 1984 she has been working as a lecturer at the Igualada Industrial Engineering Technical School - Igualada Tanning School at the Universitat Politènica de Catalunya. She is mainly involved in the development of innovative analytical methods for different industrial sectors.

Rosa Cantero Gómez obtained the degree in Chemistry at the University of Barcelona in 1989 and the Ph.D. degree in Chemistry at the Autonomous University of Barcelona. She has been a lecturer at the Igualada Engineering School, Universitat Politècnica de Catalunya (Barcelona, Spain) since 1990. In 1991 she carried out postgraduate studies on Tanning Techniques at this same School. Her research deals primarily with the application of chemometrics to control analysis in different industrial sectors. 